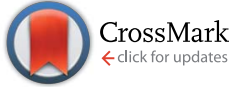

Cite this: RSC Adv., 2016, 6, 112737

DOI: 10.1039/c6ra90124g

www.rsc.org/advances

\title{
Correction: Structures, preparation and applications of titanium suboxides
}

\author{
Baoqiang $\mathrm{Xu}^{\mathrm{a}}$ Hong Yong Sohn, ${ }^{\text {b }}$ Yousef Mohassab ${ }^{\mathrm{b}}$ and Yuanpei Lan ${ }^{\mathrm{bc}}$
}

Correction for 'Structures, preparation and applications of titanium suboxides' by Baoqiang Xu et al., RSC Adv., 2016, 6, 79706-79722.

The authors regret that one of the funding bodies listed in the Acknowledgements section of the original article is incorrect. A revised version of the Acknowledgements section, in which 'the Cultivating Plan Program for the Technological Leading Talents of Yunnan Province' replaces 'the Program for Innovative Research Team in Ministry of Science and Technology of China', can be found below.

The Royal Society of Chemistry apologises for these errors and any consequent inconvenience to authors and readers.

\section{Acknowledgements}

This work was supported in part by NSF/U.S.-Egypt Joint Science and Technology Board under Grant No. IIA-1445577, National Science Foundation of China (No. 51464023), National Science Foundation of China (No. 51674129) and the Cultivating Plan Program for the Technological Leading Talents of Yunnan Province (No. 2014HA003). Baoqiang Xu expresses his special gratitude for financial support (No. 201408530020) from China Scholarship Council (CSC) for his stay as a visiting scholar at the University of Utah.

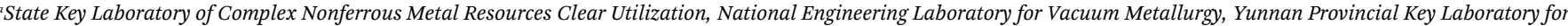
Nonferrous Vacuum Metallurgy, Kunming University of Science and Technology, Kunming 650093, P. R. China

${ }^{b}$ Department of Metallurgical Engineering, University of Utah, Salt Lake City, Utah 84112, USA. E-mail: h.y.sohn@utah.edu

${ }^{c}$ College of Material Science \& Engineering, Chongqing University, Chongqing 400044, P. R. China
} 